\title{
RELATOS DE CASOS
}

\section{PARACOCCIDIOIDOMICOSE PULMONAR ASSINTOMÁTICA E REGRESSIVA, COM POSTERIOR DISSEMINAÇÃO RELATO.DE UM CASO}

\author{
Bodo Wanke, Ezemar Marques de Andrade, José Acylino de Lima Neto e Maria \\ de Fátima Ferreira da Cruz
}

\begin{abstract}
E relatado o caso de um homem aparentemente sadio no qual uma abreugrafia de rotina evidenciou lesōes pulmonares. Enquanto se buscava a etiologia, as lesōes radiológicas foram regredindo espontaneamente até restar apenas fibrose. Por apresentar linhas de precipitação especificas frente à paracoccidioidina na prova de imunodifusão dupla em gel de agar, o paciente foi revisto 3 meses após. Nesta ocasião, persistindo a positividade da sorologia e a radiografia de tórax normal, surgiu uma lesão labial, na qual, ao exame microscópico, foi possivel demonstrar a presença do Paracoccidioides brasiliensis. Os autores ressaltam as dificuldades diagnósticas do caso e o correlacionam com as formas clínicas da doença.
\end{abstract}

Palavras chaves: Paracoccidioidomicose. Paracoccidioidomicose pulmonar. Evolução. Regressão. Disseminação.

Desde a sua descoberta em 1908 até a introdução dos compostos sulfanilamídicos no seu tratamento em $1940^{47}$, a paracoccidioidomicose era considerada doença de evolução fatal. A descrição por Fialho ${ }^{9}$, em 1946, de uma pequena lesão cicatrizada de permeio à lesões pulmonares progressivas, permitiu o raciocínio que confere à paracoccidioidomicose uma patogênese semelhante à da tuberculose e da histoplasmose. Posteriormente, lesões curadas de paracoccidioidomicose em pacientes com a doença progressiva e não submetidos à terapêutica específica, foram descritas por Fia$1 \mathrm{ho}{ }^{10} \mathrm{em}$ pulmões de 4 pacientes autopsiados e também por Motta ${ }^{30}$ em gânglio linfático de um paciente autopsiado, e, Londero ${ }^{24}$ em pulmão de um paciente pneumectomizado. Paracoccidioidomicose pulmonar primária curada (paracoccidioidomicose-infecção) foi sugerida inicialmente em pacientes com lesões pulmonares nodulares fibróticas e que apresentavam teste intradérmico positivo com a paracoccidioidina ${ }^{17}{ }^{41}$ e testes sorológicos positivos ${ }^{17}$. Contudo, a evidência inequivoca de complexo primário linfático pul-

Trabalho realizado no Instituto de Tisiologia e Pneumologia da Universidade Federal do Rio de Janeiro e na Fundação Oswaldo Cruz, Rio de Janeiro.

Correspondência para Dr. Bodo Wanke, Rua Alexandre de Gusmão, 28/201, 20520 Rio de Janeiro, RJ.

Recebido para publicação em 30/5/83. monar na paracoccidioidomicose só foi relatada duas vezes ${ }^{2}{ }^{50}$.

Apesar dos pulmões serem os órgãos mais atingidos, são as lesões extra-pulmonares que habitualmente motivam a primeira consulta ao médi$\operatorname{co}^{16} \quad 2126 \quad 2832 \quad 373940$. Estas lesões de disseminação podem ocorrer em qualquer órgão ou sistema, mas, na maioria das vezes estão situadas em pele ou mucosas. Além de ter uma exteriorização clínica muito variada, a paracoccidioidomicose habitualmente evolui por surtos ${ }^{4821} 23-2532343745$.

O caso ora relatado demonstra a importância de lembrar as micoses no diagnóstico diferencial de lesões pulmonares, e, além disso, é também um exemplo demonstrativo da dinâmica da infecção causada pelo Paracoccidioides brasiliensis.

\section{RELATO DO CASO}

Homem de 41 anos, natural da zona rural do Rio de Janeiro, onde sempre viveu. Trabalha há 20 anos em estaleiro naval de Angra dos Reis (RJ). Nunca trabalhou em lavoura. Em 20.08.81 submeteu-se a uma abreugrafia de tórax por exigência periódica rotineira da empresa onde trabalha. Neste exame foram evidenciadas "lesões com comprometimento fibroso, infiltrados intersticiais e alveolares em ambos os pulmões, poupando os ápices 
Wanke $B$, Andrade EM, Lima Neto JA, Cruz MFF. Paracoccidioidomicose pulmonar assintomática e regressiva, com posterior disseminação. Relato de um caso. Revista da Sociedade Brasileira de Medicina Tropical 16:162-167, Jul/Set, 1983

(Fig. I). O paciente, no entanto, não apresentava sintoma algum.
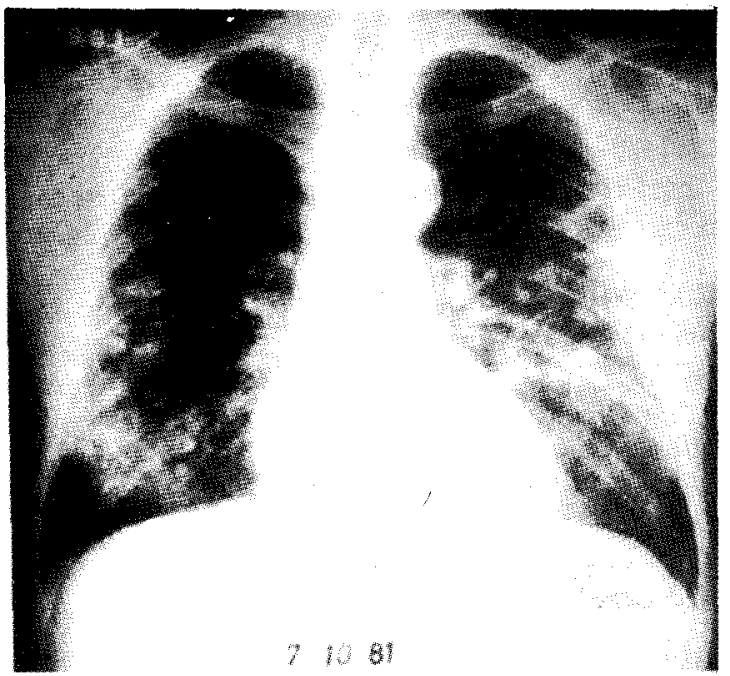

Fig. 1 - Lesões pulmonares que motivaram a internação para diagnóstico. Paciente assintomático.

Em 07.11.81 o paciente foi internado para esclarecimento diagnóstico. Nesta ocasião, nova radiografia evidenciou apreciável melhora das lesões, restando apenas fibrose. Com o diagnóstico sindromático de broncopneumonia de evolução para a cura espontânea, o paciente foi submetido a exames para esclarecer a etiologia do processo. Exames microscópicos de escarro (obtido após nebulizaçāo) foram negativos para bactérias ácido/álcool resistentes e para fungos; os cultivos de escarro fo-

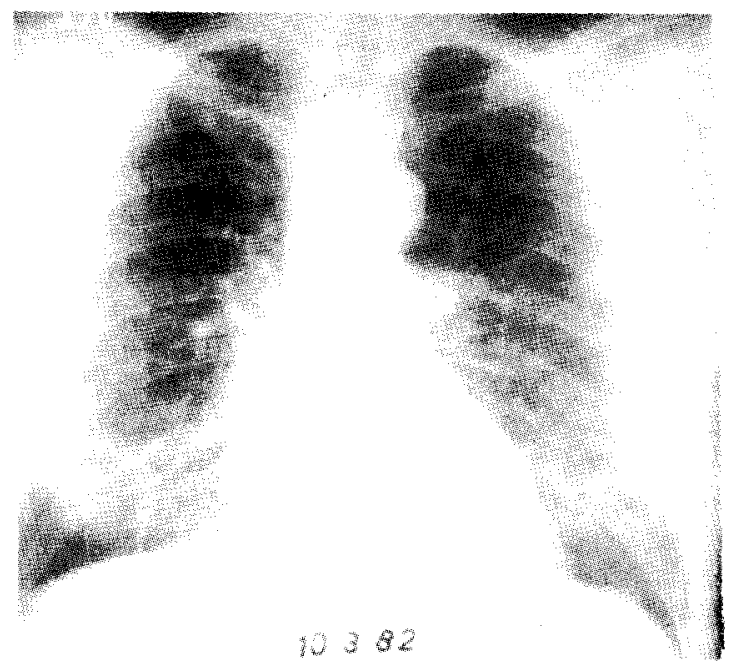

Fig. 2 - Regressão espontânea das lesōes pulmonares. Paciente com lesão labial. ram negativos para Mycobacterium e para fungos. $O$ teste intradérmico com PPD mostrou reação de $20 \mathrm{~mm}$ de diâmetro. $\mathrm{O}$ soro do paciente submetido à difusão dupla em gel de agar (DDG) foi negativo frente a histoplasmina, porém revelou dois arcos de precipitação frente à paracoccidioidina. Em virtude desta prova revelar doença em atividade, foi solicitada a reinternação do paciente.

Reinternado em 16.02.82, o paciente queixou-se de discreta dispneia aos grandes esforços. Nova radiografia de tórax foi considerada sem anormalidades, persistindo apenas traves fibróticas (Fig. 2). Três amostras de escarro (obtidas após nebulizações) e um lavado brônquio não revelaram, ao exame microscópico, fungos ou bacilos ácido/álcool resistentes. Nova prova de difusão dupla foi positiva frente à paracoccidioidina. $\mathrm{O}$ hemograma revelou 5.760 .000 hemácias $/ \mathrm{mm}^{3}$. No dia 01.03 .82 surgiram pequenas vesículas no lábio inferior à esquerda. As vesículas agrupavam-se em uma área de cerca de $1 \mathrm{~cm}$ de diâmetro. Com o diagnóstico de herpes labial pensou-se em dar alta ao paciente, porém como a DDG fosse positiva foi prevista revisão periódica. Antes de deixar o hospital verificou-se que a lesão herpetiforme do lábio havia evoluído para uma pequena ulceração de bordos elevados e de base infiltrada (Fig. 3). No material de raspagem da lesão foram encontrados elementos caracteristicos de $P$. brasiliensis (Fig. 4). Submetido a tratamento com a associação de sulfametoxazol + trimetoprim, a lesão cicatrizou rapidamente e o paciente está ativo, em excelente estado geral, sendo revisto pela última vez com 10 meses de tratamento.

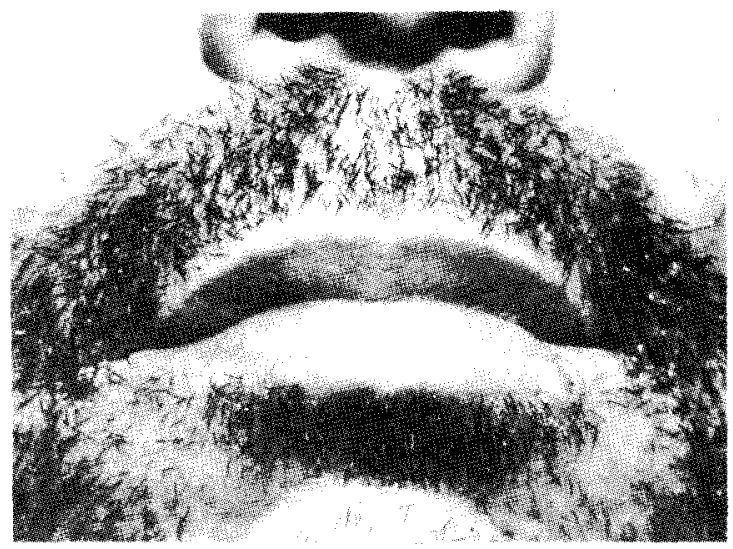

rig. 3 - Lesao do labio interior por $\boldsymbol{r}$. brasiliensis. Pulmôes "normais". 
Wanke B, Andrade EM, Lime Neto JA, Cruz MFF. Paracoccidioidomicose pulmonar assintomática e regressiva, com posterior disseminaçẫo. Relato de um caso. Revista da Sociedade Brasileira de Medicina Tropical 16:162-167, Jul/Set, 1983

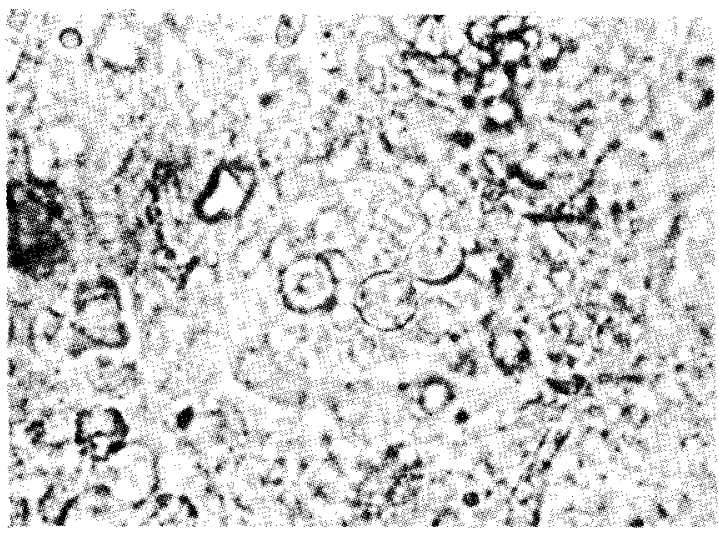

Fig. 4 - P. brasiliensis visto ao exame microscópico direto de material da lesão labial.

\section{COMENTÁRIOS}

A infecção primária pelo $P$. brasiliensis ocorre principalmente nas duas primeiras décadas da vida em residentes de áreas endêmicas ${ }^{1}{ }^{35}$ e geralmente é assintomática e regressiva, culminando com a cicatrização do processo ${ }^{14}{ }^{22}$. Em 1981 foi relatado o primeiro caso de paracoccidioidomicose pulmonar primária sintomática (aguda) em menina de $9 \operatorname{anos}^{43}$.

Nas lesōes cicatriciais de paracoccidioidomicose primária curada o $P$. brasiliensis pode estar morto ou pode permanecer viável (focos quiescentes), como o provam casos da doença descritos fora da área endêmica, muitos anos após o afastamento dos pacientes das mesmas ${ }^{3}{ }^{11}{ }^{36}$, bem como os casos de reativação de nódulos pulmonares fibróticos após o uso de terapêutica imunossupressora $^{5152}$.

\section{Por vezes a paracoccidioidomicose primária} tem curso progressivo e caráter grave, com lesões generalizadas, sobressaindo as do sistema linfáti$\operatorname{co}^{14} 202245$, caracterizando a forma disseminada juvenil ou do adulto jovem.

A forma clínica mais freqüente da paracoccidioidomicose é a progressiva do adulto, resultante da reativação de foco(s) quiescente(s) ${ }^{14}{ }^{22}{ }^{27}$. Esta forma pode ser pulmonar progressiva, quando circunscrita aos pulmões, ou disseminada, quando há lesões extra-pulmonares ${ }^{22}$.

Desde que Haberfeld ${ }^{15}$ relatou em 1919 um caso de paracoccidioidomicose de localização ex- clusivamente pulmonar, as lesões paracoccidioidicas pulmonares têm merecido mais atenção ${ }^{4} 5910$

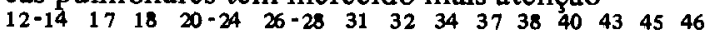
48-52. É muito variável a sintomatologia respiratória da paracoccidioidomicose pulmonar. Usualmente os pacientes referem história de longos períodos - meses ou anos - apresentando sintomas de infecção respiratória, rotulados de "gripes" recorren-

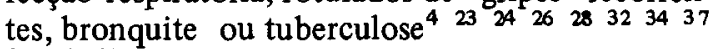
${ }^{45} 4648$. Por vezes os sintomas são representados apenas por progressivos cansaco físico e dispnéia de esforços ${ }^{23} 242832374548$. Há casos, porém, em que a doença tem curso assintomático e o diagnóstico constitui achado de surpresa, por ocasião de exame radiológico do tórax ${ }^{3} 26 \quad 28 \quad 3233 \quad 3648$, tal como ocorreu no caso que ora relatamos.

Quando as lesões pulmonares são assintomáticas ou quando são acompanhadas de sintomas inexpressivos, geralmente o paciente só procura cuidados médicos após o surgimento de uma lesão de disseminação. Tal ocorreria com o nosso paciente, não houvesse ele sido submetido à abregrafia $\mathrm{e}$ posterior sorologia. Da mesma forma, tal como em nosso paciente, a lesão de disseminação mais freqüentemente se localiza nas mucosas e decorre de surto de disseminação hematogênica do $P$. brasiliensis $^{27}$. É lamentável que o diagnóstico da micose freqüentemente seja feito apenas nesta etapa de sua evolução, pois nesta fase avançada é freqüente e, por vezes, importante, o comprometimento fi-

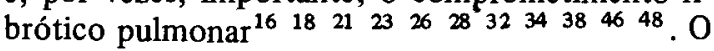
caso ora relatado ilustra muito bem a dinâmica da infecção pelo $P$. brasiliensis, mostrando que o reconhecimento da micose antes do aparecimento da lesão tegumentar só ocorreu por acaso. Além disso, quando a lesão mucosa surgiu em nosso paciente, os pulmões eram radiologicamente normais. Tal fato tem sido verificado em pacientes com lesões extrapulmonares nos quais a radiografia do tórax é dada como normal, porém nos quais é demonstrada a existência de lesão do pulmão pelo achado do $P$. brasiliensis no escarro ou no lavado brônquico ${ }^{45}$, em biópsia pulmonar ${ }^{26} 45$ ou após

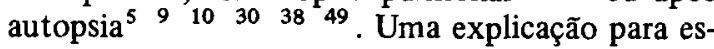
ta "normalidade" pulmonar radiológica nesses casos pode ser devida à predominância de lesões granulomatosas pequenas, sem tamanho suficiente para serem visualizáveis radiologicamente. Tais lesð̋es, muitas vezes associadas a lesões fibróticas de regressão espontânea, foram descritas inicialmente por Fialho $0^{9} 10$ e posteriormente confirmadas por outros $^{5}{ }^{49}$. Por outro lado, Londero e Severo ${ }^{24}$ descreveram sumariamente caso (no 11) semelhante ao que ora relatamos. Ademais, Restrepo e 
Wanke B, Andrade EM, Lima Neto JA, Cruz MFF. Paracoccidioidomicose pulmonar assintomática e regressiva, com posterior disseminação. Relato de um caso. Revista da Sociedade Brasileira de Medicina Tropical 16:162-167, Jul/Set, 1983

cols. ${ }^{45}$ no caso B-2a relatam a observação da melhora espontânea de lesões radiológicas em paciente com paracoccidioidomicose.

A lesão tegumentar de nosso paciente inicialmente mimetizava clínica e topograficamente herpes labial. A evolução desta lesão, porém, não correspondia à de uma lesão herpética. Machado Filho e Miranda ${ }^{25}$ em 1960 já refiriram a observação de "lesర̋es iniciais que surgiram como minúsculas flictenas" e Castro e cols. ${ }^{6}$ citam a verificação de lesões vesiculosas da pele, inicialmente diagnosticadas como varicela. Cabe ponderar que estas lesões são nitidamente de origem endógena ${ }^{27}$ e não correspondem a um inóculo traumático local ${ }^{42}$. $O$ único caso de indiscutível inoculação traumática cutânea foi publicado por Castro e cols. ?

Para finalizar deve ser destacado o valor da sorologia, representada pela prova de difusão dupla em gel de agar. A DDG é prova de grande utilidade para a triagem de pacientes que apresentam lesões radiológicas pulmonares em que outros diagnósticos foram excluídos. Sendo reação simples, específica e de alta sensibilidade ${ }^{19} 44$, merece ser usada na rotina.

\section{SUMMARY}

A case of spontaneously healed pulmonary paracoccidioidomycosis in an apparently healthy man is reported. At first the diagnosis was based on chest X-ray lesions and a positive precipitation test. Three months later, despite the persisting normal chest $X$-ray, diagnosis was confirmed by the finding of Paracoccidioides brasiliensis on a lip lesion.

The authors emphasize that diagnosis was rather difficult - because the patient remained symptomless - and to correlate this case with the clinical forms of the disease.

Key words: Paracoccidioidomycosis. South American blastomycosis. Lung involvement.

\section{AGRADECIMENTO}

Ao Prof. A. T. Londero pelas sugestões e revisão do texto.

\section{REFERÊNCIAS BIBLIOGRĂFICAS}

1. Albornoz MCB, Albornoz RM. Estudio de la sensibilidad especifica en residents de un area endemica a la paracoccidioidomicosis en Venezuela. Mycopathologia 45:65-75, 1971.

2. Angulo-Ortega A. Calcifications in paracoccidioidomycosis: are they the morphological manifestation of subclinical infection? In: Paracoccidioidomycosis. PAHO Sc. Pub. no 254. Pan American Health Organization, Washington, p. 129-133, 1972.

3. Balabanov K, Balabanoff VA, Angelov N. Blastomycose Sud-Américaine chez un laboureur bulgare revenu depuis 30 ans de Brésil. Mycopathologia 24:265$-270,1964$.

4. Borrero J, Restrepo A, Robledo M. Blastomicosis suramericana de forma pulmonar pura. Antioquia Médica 15:503-516, 1965.

5. Brass K. Observaciones sobre la anatomia patológica, patogenia y evolución de la paracoccidioidomicosis. Mycopathologia 37: 119-138, 1969.

6. Castro RM, Del Negro G, Bassoi ON, Faria CV. Dificuldades diagnósticas na blastomicose sul-americana. Revista Paulista de Medicina 53:479-496, 1958.

7. Castro RM, Cucé LC, Fava Netto C. Paracoccidioidomicose. Inoculação acidental "in anima nobile". Relato de um caso. Medicina Cutânea Ibero Latino-Americana 4: 289-292, 1975.

8. Del Negro G. Paracoccidioidomicose (blastomicose sul americana). Aspectos clínicos. Ars Curandi $7: 30-36,1975$.

9. Fialho A. Localizações pulmonares da "Micose de Lutz". Anatomia patológica e patogênese. Tese. Faculdade de Medicina da Universidade Federal do Rio de Janeiro, Rio de Janeiro, 1946.

10. Fialho A. Patogenia da blastomicose sul-americana. Revista Brasileira de Tuberculose 24:97-117, 1956.

11. Fountain FF, Sutliff WD. Paracoccidioidomycosis in the United States. American Review of Respiratory Disease 99: 89-93, 1969.

12. Furtado T. Comprometimento pulmonar na blastomicose sul-americana. Revista da Associação Médica de Minas Gerais 3:49-57, 1952.

13. Furtado T. Infection versus disease in paracoccidioidomycosis. In: Paracoccidioidomycosis. PAHO Sc. Pub. n? 254. Pan American Health Organization, Washington, p. 271-277, 1972.

14. Giraldo R, Restrepo A, Gutiêrrez F, Robledo $M$. Londoño F, Hernández H, Sierra F, Calle G. Pathogenesis of paracoccidioidomycosis. A model based on the study of 46 patients. Mycopathologia 58:63$-70,1976$. 
Wanke B, Andrade EM, Lima Neto JA, Cruz MFF. Paracoccidioidomicose pulmonar assintomática e regressiva, com posterior disseminação. Relato de um caso. Revista da Sociedade Brasileira de Medicina Tropical 16:162-167, Jul/Set, 1983

15. Haberfeld W. Blastomicose. Nova contribuição ao estudo da blastomicose interna. Revista Médica de São Paulo 3: 5-7, 1919.

16. Lacaz CS. Micologia médica. 6ạ ed. Sarvier, São Paulo, 1978.

17. Lacaz CS, Passos Filho MCR, Fava Netto C, Macarron B. Contribuição para o estudo da "blastomicose-infecção". Inquérito com a paracoccidioidina. Estudo sorológico e clínico-radiológico dos paracoccidioidino-positivos. Revista do Instituto de Medicina Tropical de São Paulo 1:245-259, 1959.

18. Londero AT. Aspectos de la blastomicosis sudamericana en Rio Grande do Sul, Brasil. El Torax 17:56$-59,1968$.

19. Londero AT, Lopes JOS, Ramos CD, Severo LC. A prova da dupla difusão em gel de agar no diagnóstico da paracoccidioidomicose. Revista da Associação Médica do Rio Grande do Sul 25:272-275, 1981.

20. Londero AT, Melo IS. Paracoccidioidomycosis in childhood. A critical review. Mycopathologia (em publicação).

21. Londero AT, Ramos CD. Paracoccidioidomycosis. A clinical and mycological study of forty-one cases observed in Santa Maria, RS, Brasil. American Journal of Medicine 52:771-775, 1972.

22. Londero AT, Ramos CD, Lopes JO. Paracoccidioidomicose: classificação das formas clínicas. Revista Uruguaia de Patologia Clínica y Micobiologia 14:3-9, 1976.

23. Londero AT, Ramos CD, Lopes JOS. Progressive pulmonary paracoccidioidomycosis. A study of 34 cases observed in Rio Grande do Sul (Brasil). Mycopathologia $63: 53-56,1978$.

24. Londero AT, Severo LC. The gamut of progressive pulmonary paracoccidioidomycosis. Mycopathologia 75:65-74, 1981.

25. Machado Filho J, Miranda JL. Considerações relativas à blastomicose sul-americana. Localizações, sintomas iniciais, vias de penetração e disseminação em 313 casos consecutivos. Hospital 58:99-137, 1960.

26. Machado Filho J, Miranda JL. Considerações relativas à blastomicose sul-americana. Da participação pulmonar entre 338 casos consecutivos. Hospital 58:431-449, 1960.

27. Mackinnon JE. Pathogenesis of South American blastomycosis. Transactions of the Royal Society of Tropical Medicine and Hygiene 53:487-494, 1959.
28. Mackinnon JE. On the importance of South American blastomycosis. Mycopathologia 41:187-193, 1970.

29. Melo IS, Londero AT. Spontaneously regressive pulmonary lesions in paracoccidioidomycosis. Case report and review. Mycopathologia (aceito para publicação).

30. Motta LC. Granulomatose paracoccidióidica: Forma orgânica isolada. Revista do Hospital de Clínicas da Faculdade de Medicina da Universidade de São Paulo $11: 353-361,1956$.

31. Negroni P, Negroni R. Nuestra experiencia de la blastomicosis sudamericana en la Argentina. Mycopathologia 26:264-272, 1965.

32. Passos Filho MCR. Blastomicose sul-americana. Comentários em torno de 83 casos de localização pulmonar. Classificação radiológica. Hospital 70: 109$134,1966$.

33. Passos Filho MCR, Nahas L. Tratamento da blastomicose sul-americana de localização pulmonar pela 6 sulfanilamido - 2,4 - dimetilpirimidina. Aspectos do diagnóstico e da evolução clínica. Hospital 55: 237-263, 1959.

34. Paula A. O pulmão da blastomicose brasileira. Jornal Brasileiro de Medicina 6:480-483, 1962.

35. Pedrosa PN. Paracoccidioidomicose. Inquérito intradérmico com paracoccidioidina em zona rural do Estado do Rio de Janeiro. Tese de Mestrado. Faculdade de Medicina da Universidade Federal do Rio de Janeiro, Rio de Janeiro, 1976.

36. Perry OH Weed AL, Kierland RR. South American blastomycosis. Report of a case and review of laboratory features. Archives of Dermatology 70:477-482, 1954.

37. Pinto HB. La paracoccidioidosis brasiliensis como enfermedad sistemica. Comentários de la casuistica venezolana. Mycopathologia 15:90-114, 1961.

38. Pinto Lima FX. Contribuição ao estudo clínico e radiológico da blastómicose pulmonar. Departamento de Publicações da Escola Politécnica de São Paulo, SP, 1952.

39. Porto JA. Blastomicose sul-americana (lesão anal). Tribuna Médica 350:17-18, 1969.

40. Porto JA, Silva JR. Micose de Lutz (blastomicose sul-americana): consideraçōes a propósito da manifestação inicial e do comprometimento visceral. Jornal Brasileiro de Medicina 6: 192-203, 1962. 
41. Posada H. Encuesta sobre la tuberculosis, la histoplasmosis y la paracoccidioidomicosis en un hospital de tuberculose. Antioquia Médica 18:49-57, 1968.

42. Proença NG, Castro RM, Alonso FF. Curso benigno de paracoccidioidomicose de possível inoculação cu-. tânea. Revista da Associação Médica Brasileira 27: 170-172, 1981.

43. Ramos CD, Londero AT, Gal MCL. Pulmonary paracoccidioidomycosis in a nine year old girl. Mycopathologia $74: 15-18,1981$.

44. Restrepo A, Moncada LH. Serologic procedures in the diagnosis of paracoccidioidomycosis. In: Proceedings, International Symposium on Mycoses. PAHO Sc. Pub. no 205. Pan American Health Organization, Washington. p. 101-111, 1970.

45. Resprepo A, Robledo M, Giraldo R, Hernández $H$, Sierra F, Gutiérrez F, Londoño F, Lopez R, Calle G. The gamut of paracoccidioidomycosis. American Journal of Medicine 61:33-42, 1976.

46. Restrepo A, Robledo M, Gutiérrez F, Sanclemente M, Cantañeda E, Calle G. Paracoccidioidomycosis (South American blastomycosis). A study of 39 cases observed in Medellín, Colombia. American Journal of Tropical Medicine and Hygiene 19:68-76, 1970.
47. Ribeiro DO. Nova terapêutica para a blastomicose. Publicações Médicas (S. Paulo) 12:36-54, 1940.

48. Rodríguez C, Rincón NL, García GT. Contribucion al estudio de la paracoccidioidomicosis brasiliensis en Venezuela. Consideraciones sobre 62 casos estudiados con especial referencia a las localizaciones respiratórias. Mycopathologia 15: 115-138, 1961.

49. Salfelder K, Doehnert G, Doehnert HR. Paracoccidioidomycosis. Anatomic study with complete autopsies. Virchos Archives (Pathologia Anatomy) 348:51-76, 1976.

50. Severo LC, Geyer GR, Londero AT, Porto NS, Rizzon CFC. The primary pulmonary lymph node complex in paracoccidioidomycosis. Mycopathologia 67:115-118, 1979.

51. Severo LC, Palombini BC, Utz E, Braun SN. Paracoccidioidomicose pulmonar resultante de lesão quiescente em paciente imunossuprimido. Jornal de Pneumologia 6: 21-22, 1980.

52. Wanke B. Paracoccidioidomicose. Inquérito intradérmico com paracoccidioidina em zona urbana do Município do Rio de Janeiro. Tese de Mestrado. Faculdade de Medicina da Universidade Federal do Rio de Janeiro, Rio de Janeiro, p. 16-17, 1976. 